\title{
Predictors of Mortality for Delirium in Palliative Care
}

\author{
Meera R. Agar, MBBS, MPC, PhD, FAChPM, FRACP, ${ }^{1-4}$ \\ Stephen J. Quinn, B Math, M. Math, PhD, ${ }^{5}$ \\ Gregory B. Crawford, MBBS, MPHC, MD, FRACGP, FAChPM, ${ }^{6,7}$ \\ Christine S. Ritchie, MD, MSPH, \\ Jane L. Phillips, RN, PhD, ${ }^{3}$ \\ Aileen Collier, RN, PhD, ${ }^{1}$ \\ David C. Currow, BMed, MPH, PhD, FRACP, FAHMS ${ }^{1,5}$
}

\begin{abstract}
Introduction: Delirium has a high mortality rate. Understanding predictors of prognosis in patients with delirium will aid treatment decisions and communication. This study aimed to explore variables associated with death during an established episode of delirium in palliative care when haloperidol treatment had been commenced.

Methods: A consecutive cohort of palliative care patients, from 14 centers across four countries, is reported. The outcome of interest was death within 14 days from commencement of haloperidol treatment for delirium. Clinicodemographic variables explored were delirium severity, age, gender, primary life limiting illness, body mass index (BMI), total daily haloperidol dose at baseline ( $\mathrm{mg}$ ), functional status, and comorbidities.

Results: One hundred and sixteen palliative care patients where vital status was known were included in the analysis; $45 \%(n=52)$ died within 10 days, and 56\% $(n=65)$ died within 14 days. In multivariate analyses no clinical or demographic variables predicted death, apart from lower BMI in noncancer patients.

Conclusion: This study has shown a very high mortality rate within two weeks of commencing haloperidol for delirium in palliative care, with no clear clinical predictors for those with a higher chance of dying. Having a higher BMI offered some benefit in survival, but only in noncancer patients. When delirium occurs in advanced illness, discussion should be initiated about the gravity of the clinical situation.
\end{abstract}

\section{Introduction}

DELIRIUM IS FREQUENTLY encountered in palliative care populations, with prevalence rates of $13 \%-42 \%$ cited,

and is associated with significant distress for patients, families, and health professionals. ${ }^{1,2}$ Delirium occurrence in palliative care should inform treatment discussions with patients and families, ${ }^{2}$ including the possibility that delirium may be a specific prelude to death.

Delirium outcomes in older adults have been extensively explored, and it is associated with accelerated functional and cognitive decline, longer hospital stays, risk of institutionalization, and higher risk of death. ${ }^{3-11}$ The National Institute for Health Care Excellence (NICE) delirium guidelines ${ }^{12}$ reported an inhospital mortality odds ratio (OR) of 2.6 (95\% CI 0.7-6.2) and mortality at one month of 3.0 (95\% CI 1.1-8.4) in medical, surgical, orthopedic, and intensive care, but explicitly excluded palliative and cancer settings. ${ }^{13-15}$

Similarly, in palliative care populations, increased mortality associated with delirium has been confirmed. ${ }^{16-19}$ Three prognostic scores in advanced illness include delirium as a predictor of mortality, namely the palliative prognostic index (PPI), palliative prognostic score (PaP), and In-hospital Mortality Prediction in Advanced Cancer Patients (IMPACT). ${ }^{20-23}$ In the PPI, presence of delirium at a single time point, without accounting for duration or severity of delirium, had a sensitivity of $80 \%$ and specificity of $85 \%$ in predicting survival in advanced cancer. ${ }^{22}$ When a delirium diagnosis is added to the $\mathrm{PaP},{ }^{20}$ it is an independent predictor of survival in advanced cancer, with a median survival of 21 days (95\% CI 16-27)

\footnotetext{
${ }^{1}$ Discipline of Palliative and Supportive Services, Flinders University, Adelaide, South Australia.

${ }^{2}$ South West Sydney Clinical School, University of New South Wales, Sydney Australia.

${ }^{3}$ Centre for Cardiovascular and Chronic Care, Faculty of Health, University of Technology Sydney, Ultimo, Australia.

${ }^{4}$ Ingham Institute of Applied Medical Research, Sydney, Australia.

${ }^{5}$ Flinders Clinical Effectiveness, Flinders University, Adelaide, Australia.

${ }^{6}$ Discipline of Medicine, University of Adelaide, Adelaide, Australia.

${ }^{7}$ Northern Adelaide Local Health Network, Adelaide, Australia.

${ }^{8}$ Department of Medicine, Division of Geriatrics, University of California San Francisco, San Francisco, California.

${ }^{9}$ The Jewish Home of San Francisco, San Francisco, California.

Accepted May 10, 2016.
} 
compared to 39 days (95\% CI 33-49) for people without delirium, and slightly improves its predictive value. ${ }^{23}$ More recently, IMPACT was developed to explore utility of clinical variables to predict mortality during acute palliative care unit admissions, ${ }^{21}$ with mortality independently associated with physician-diagnosed delirium (on DSM-IV-R criteria; $\mathrm{OR}=4.3$, 95\% CI $1.3-14.8 ; p=0.02) .^{21}$

Once delirium has occurred, mortality is predicted by illness severity, ${ }^{24}$ age, and prior cognitive impairment/dementia in older adults. ${ }^{4}$ Two studies to date have demonstrated that shorter survival for palliative patients with delirium is associated with persistent delirium, severe cognitive impairment related to the delirium episode, both younger and older age, hypoxemia, and metabolic changes related to organ failure. ${ }^{17,18}$ In previous studies of patients with cancer, dementia was only present in $7 \%$ of patients with delirium, a major predictor of higher mortality due to delirium in other settings. ${ }^{17}$ The purpose of this study was to explore predictors of mortality associated with delirium in palliative care.

\section{Methods}

This was a secondary analysis of a consecutive prospective cohort of hospice/palliative care patients prescribed haloperidol for delirium as part of routine clinical practice in 14 centers in four countries. ${ }^{25,26}$ Participating sites had ethical approval under low or negligible risk research or ethical waivers. ${ }^{25,26}$ The National Cancer Institute's Common Terminology Criteria for Adverse Events (NCI CTCAE) ${ }^{27}$ was used to grade delirium severity (Table 1).

Baseline clinical variables were derived from routinely collected and noninvasive clinical data and included age, gender, life limiting illness (cancer, noncancer), body mass index (BMI), daily haloperidol dose at baseline (mg), functional status (Australian-modified Karnofsky Performance Status scale [AKPS]), ${ }^{28}$ and comorbidities (Charlson Comorbidity Index [CCI] while removing the score for the primary life-limiting illness). ${ }^{29,30}$

Death at 14 days from commencing haloperidol was the primary outcome, given that this was the time period covered by prospective data collection, encompassing the time frame for the immediate and short-term benefits and harms of haloperidol in treating delirium.

Delirium severity was divided into three groups according to NCI CTCAE scores: mild (score 1), moderate (score of 2), and severe (score of 3 or 4 , where hospitalization was required). Differences in continuous demographic attributes

\section{Table 1. National Cancer Institute's Common Terminology Criteria fOR Adverse Events ${ }^{27}$ Graded Delirium SeVerity}

\footnotetext{
$0 \quad$ No delirium

1 Mild acute confusional state

2 Moderate and acute confusional state limiting instrumental activities of daily living

3 Severe and acute confusional state limiting self-care activities of daily living with hospitalization indicated

4 Life-threatening consequences, threats of self-harm or harm to others, hospitalization indicated

5 Death
}

between groups were assessed by analysis of variance or the Kruskal-Wallis test, depending on whether normality conditions were met. For categorical variables, chi-square tests were used. Cox proportion hazard regression analysis was conducted to adjust for potential confounders identified in the literature that may help to predict death: gender, age, functional status, primary illness, the number of comorbid conditions, BMI, 24-hour haloperidol dose, and baseline delirium severity. A $p$-value of less than 0.05 (two-tailed) was considered statistically significant, and results are presented as hazard ratios (HR) with $95 \%$ confidence intervals. All analyses were conducted using Stata 13.1 (StataCorp, Texas, USA).

\section{Results}

These patients $(n=119)$ have been described previously and had a median age of 75 years (range 34-99) and the majority $(88 \%)$ had cancer. ${ }^{25}$ For this substudy, patients were stratified by baseline delirium severity (Table 2 ). Where vital status was known, at 14 days, 65 of the 116 (56\%) patients had died. Of the patients who died, care was being delivered in a palliative care unit $(n=21)$, acute care palliative care service $(n=9)$, and community palliative care service $(n=23)$, with the remainder of the participants from services that had both community and inpatient services. The mean age in the moderate ( $n=37 ; 77$ years) and severe delirium ( $n=50 ; 75$ years) categories was older than the mild category $(n=27 ; 66$ years; $p<0.002)$.

Severity of delirium, age, gender, haloperidol dose, functional status, or presence of comorbidities was not a predictor of death after adjusted for the other available confounders known potentially to influence delirium outcomes (Table 3). The only finding was a significant interaction between BMI and primary illness $(p=0.036)$. In noncancer patients $(n=14)$, higher BMI was associated with decreasing risk of death $(\mathrm{HR}=0.66$; $(95 \%$ CI $0.45-0.97 ; p=0.033)$, but this association was not seen in majority of the participants who were cancer patients $(n=105)(p=0.62)$.

\section{Discussion}

This study has found that lower BMI was the only factor predictive of poorer survival for palliative care patients with delirium receiving treatment with haloperidol, but only in $12 \%$ of the sample who had noncancer diagnoses. Our findings also confirm a high immediate mortality rate for delirium, ${ }^{16-18}$ in a predominantly cancer palliative care population with delirium receiving haloperidol, consistent with prior studies. ${ }^{22}$ Our data have not demonstrated an association with age, where prior studies have shown contradictory results with both younger and older age groups seemingly at risk. ${ }^{4,17,18}$ Our study chose to include only measures, which were routinely collected in clinical practice and readily evaluated at the bedside without invasive investigations. We cannot make direct comparisons between associations with the physiological parameters and formal delirium or cognitive measures used in two prior studies that explored predictors of mortality in palliative care. ${ }^{17,18}$ Both studies demonstrated shorter survival with persisting or irreversible delirium. ${ }^{17,18}$ The first study explored a cohort of cancer patients without central nervous system involvement $(n=140)$ and demonstrated a $25 \% 30$-day mortality, with younger age, hypoxemia, 
Table 2. Demographics of the Analysis Set by Delirium Severity

\begin{tabular}{|c|c|c|c|c|}
\hline & Mild $(\mathrm{n}=28)$ & Moderate $(\mathrm{n}=40)$ & Severe $(\mathrm{n}=51)$ & $\mathrm{p}^{\mathrm{a}}$ \\
\hline Female gender, $n(\%)$ & $12(42.9)$ & $13(32.5)$ & $23(46.0)$ & 0.42 \\
\hline Age mean (SD) & $66.3(13.0)$ & 76.5 (10.6) & 74.5 (12.9) & 0.003 \\
\hline AKPS (median, LQ-UQ) & $40(30-40)$ & $30(20-40)$ & $30(20-40)$ & 0.07 \\
\hline Comorbidities (median, LQ-UQ) Charos Charlson & $2(1-2)$ & $2(1-2)$ & $2(1-3)$ & 0.94 \\
\hline Haloperidol dose (median, LQ-UQ) & $2(1-2)$ & $1.25(1-2)$ & $2(1-2)$ & 0.17 \\
\hline BMI (mean, SD) & $25.1(4.5)$ & $24.5(4.3)$ & $24.7(5.1)$ & 0.91 \\
\hline Primary life limiti & $27(96.4)$ & $36(90.0)$ & $42(82.4)$ & 0.16 \\
\hline Number who had died by 14 days, $n(\%)$ & $12(42.9)$ & $18(45.0)$ & $28(54.9)$ & 0.50 \\
\hline
\end{tabular}

${ }^{a}$ Difference between groups using ANOVA, Kruskal-Wallis, or chi-square tests as appropriate.

AKPS, Australia-modified Karnofsky Performance Status; BMI, body mass index; LQ, lower quartile; SD, standard deviation; UQ, upper quartile

and organ impairment (defined as increased serum urea nitrogen levels and increased serum alkaline phosphatase) predictive of the patients who were more likely to die. ${ }^{18}$ By contrast, predictors of mortality in a consecutive cohort of palliative care inpatients $(n=121)$ who were followed until recovery or death included the following: severe cognitive changes due to the delirium (on Cognitive Test for Delirium), increasing age, and organ failure (on Delirium Etiology Checklist). ${ }^{17}$ In the second study, only $13 \%$ had prior cognitive impairment, a major predictor of higher mortality due to delirium in other settings. ${ }^{17}$

With a median survival of less than two weeks, the real challenge for clinical practice is what conversations with families the diagnosis of delirium should generate. These findings suggest that delirium occurrence by itself should prompt open discussions with the person and his/her family about the likely prognosis associated with delirium in the context of advanced disease, as studies to date have not defined any consistent clinicodemographic factors that predict risk of death.

The limitations of this study include lack of formal measurement of prior cognitive impairment and liver and renal

Table 3. Associations Between Death and Predictors

\begin{tabular}{lcc}
\hline & Univariable & Multivariable \\
\hline $\begin{array}{l}\text { Death } \\
\text { Delirium }\end{array} \quad$ OR $(95 \% \mathrm{CI})$ & OR $(95 \% \mathrm{CI})$ \\
$\quad \begin{array}{c}\text { Mild } \\
\quad \text { (reference group) }\end{array}$ & 0 & 0 \\
$\quad$ Moderate & $1.25(0.62-2.52)$ & $0.82(0.34-1.97)$ \\
$\quad$ High & $1.55(0.79-3.02)$ & $1.41(0.60-3.32)$ \\
Female gender & $0.98(0.59-1.62)$ & $0.95(0.53-1.70)$ \\
Age & $1.01(0.99-1.03)$ & $1.00(0.97-1.03)$ \\
AKPS & $0.99(0.97-1.01)$ & $0.98(0.95-1.01)$ \\
Comorbidities & $0.81(0.65-1.01)$ & $0.87(0.65-1.15)$ \\
Haloperidol dose & $0.98(0.83-1.16)$ & $0.86(0.70-1.06)$ \\
BMI & & \\
$\quad$ Noncancer primary & $0.86(0.65-1.14)$ & $\mathbf{0 . 6 7}(\mathbf{0 . 4 6 , 0 . 9 7})$ \\
$\quad$ life limiting illness & & \\
$\quad$ limiting illness & $1.02(0.96-1.08)$ & $1.02(0.95-1.09)$ \\
Primary illness & & \\
$\quad$ For average BMI & $0.85(0.27-2.71)$ & $2.78(0.48-16.01)$ \\
\hline
\end{tabular}

OR, odds ratio. Significant association in bold. function not routinely measured in the participating services, restricting comparisons with predictors of mortality used in other studies. The participants predominantly had cancer, limiting generalizability to noncancer patients; and almost half of the participants were in the inpatient setting, which may reflect a group who were sicker or had more complex medical problems. Delirium and its severity were assessed by clinicians, and no specific delirium severity scale or method was used to diagnose delirium; however, the grading categories utilized have face validity, reflecting the clinical issues that are deemed serious, namely functional decline, hospitalization, and patient safety. It is possible that hypoactive delirium, the more prevalent subtype in palliative care with poorer outcomes, was underrepresented, either because patients did not present with symptoms deemed to require treatment with haloperidol or the clinician did not utilize comprehensive assessments methods to detect this subtype of delirium that is more subtle in presentation. ${ }^{31-33}$

The predictors of survival may differ in those with delirium not treated with haloperidol. In this study, survival time was determined from the time of commencement of haloperidol, and there may be other factors influencing when such treatment is started after delirium occurs. Antipsychotic treatment has been demonstrated in other populations to be associated with increased mortality. ${ }^{34}$ The delirium subtype or phenomenology, which may motivate clinicians to treat delirium with antipsychotics, has been associated with differing mortality outcomes. ${ }^{35}$ Equally the choice of other antipsychotics other than haloperidol may be related to specific clinical scenarios with different mortality, for example, refractory delirium in the imminently dying patient.

Ultimately, delirium in advanced disease carries a very poor prognosis. The current work needs to be expanded to a much larger prospective cohort and consent participants to measure physiological variables and assess delirium phenomenology in more detail, to better understand which patient or treatment characteristics contribute most to poorer outcomes and to serve as a guide for clinical conversations. Evaluation of potential preventative interventions to reduce the incidence of delirium in this population and assess impact on survival is also urgently needed.

\section{Acknowledgments}

Thanks go to all the clinicians and the clinical units who have contributed to this program, Mr. Zac Vandersman and Ms. Caroline Litster for data entry and coding. Participating 
units include Sunshine Coast Hospital \& Health Service, Braeside Hospital, Calvary Mater Newcastle, Sacred Heart Hospice, St. Vincent's Sydney, Calvary Healthcare, Greenwich Hospital, Wolper Private Hospital, Peter MacCallum Cancer Institute, St. Vincent's Hospital (Melbourne), Barwon Health, Royal Melbourne Hospital, Calvary Hospital North Adelaide, Southern Adelaide Palliative Services, Grey Nuns Hospital Edmonton, Canada, Leeds Teaching Hospitals NHS Trust, England UK, and Haven of Hope Hospital, Hong Kong. The Australian Palliative Care Clinical Studies Collaborative is supported by funding from the Palliative Care Branch of the Australian Government's Department of Health and Ageing.

\section{Author Disclosure Statement}

No competing financial interests exist.

\section{References}

1. Hosie A, Davidson PM, Agar M, et al.: Delirium prevalence, incidence, and implications for screening in specialist palliative care inpatient settings: A systematic review. Palliat Med 2013;27:486-498.

2. Breitbart W, Alici Y, Breitbart W, et al.: Agitation and delirium at the end of life: "We couldn't manage him". JAMA 2008;300:2898-2910.

3. Pitkala KH, Laurila JV, Strandberg TE, et al.: Prognostic significance of delirium in frail older people. Dement Geriatr Cogn Disord 2005;19:158-163.

4. McCusker J, Cole M, Abrahamowicz M, et al.: Delirium predicts 12-month mortality. Arch Intern Med 2002;162: 457-463.

5. Thomas RI, Cameron DJ, Fahs MC.: A prospective study of delirium and prolonged hospital stay. Exploratory study. Arch Gen Psychiatry 1988;45:937-940.

6. Pompei P, Foreman M, Rudberg MA, et al.: Delirium in hospitalised older persons: Outcome and predicotrs. $\mathrm{J}$ Am Geriatr Soc 1994;42:809-815.

7. Kakuma R, Du fort GG, Arsenault L, et al.: Delirium in older emrgency department patients discharged home: Effects on survival. J Am Geriatr Soc 2003;51:443-450.

8. Ely EW, Gautam S, Margolin R, et al.: The impact of delirium in the intensive care unit on hospital length of stay. Intensive Care Med 2001;27:1892-1900.

9. Marcantonio ER, Flacker JM, Michaels M, et al.: Delirium is independently associated with poor functional recovery after hip fracture. J Am Geriatr Soc 2000;48:618-624.

10. Murray AM, Levkoff SE, Wetle T, et al.: Acute delirium and functional decline in the hospitalised elderly. $\mathrm{J}$ Gerontol 1993;48:M181-M186.

11. Gustafson Y, Berggren D, Brannstrom B, et al.: Acute confusional states in elderly patients treated for femoral neck fracture. J Am Geriatr Soc 1988;36:525-530.

12. National Institute for Health and Clinical Excellence (NICE) National Clinical Guideline centre: Delirium: Diagnosis, prevention and management. Secondary Delirium: Diagnosis, prevention and management 2010. www.nice. org.uk/nicemedia/live/13060/49908/49908.pdf. (Last accessed September 15, 2015).

13. Inouye SK, Rushing JT, Foreman MD, et al.: Does delirium contribute to poor hospital outcomes? A three-site epidemiologic study. J Gen Inter Med 1998;13:234-242.
14. Leslie DL, Zhang Y, Holford TR, et al.: Premature death associated with delirium at 1-year follow-up. Arch Intern Med 2005;165:1657-1662.

15. Marcantonio E, Ta T, Duthie E, et al.: Delirium severity and psychomotor types: Their relationship with outcomes after hip fracture repair. J Am Geriatr Soc 2002;50: $850-857$.

16. Lawlor PG, Gagnon B, Mancini IL, et al.: Occurrence, causes, and outcome of delirium in patients with advanced cancer: A prospective study. Arch Intern Med 2000;160: 786-794.

17. Leonard M, Raju B, Conroy M, et al.: Reversibility of delirium in terminally ill patients and predictors of mortality. Palliat Med 2008;22:848-854.

18. Tuma R, DeAngelis LM: Altered mental status in patients with cancer. Arch Neurol 2000;57:1727-1731.

19. Vigano A, Dorgan M, Buckingham J, et al.: Survival prediction in terminal cancer patients: A systematic review of the literature. Palliat Med 2001;14:363-374.

20. Caraceni A, Nanni O, Maltoni M, et al.: Impact of delirium on the short term prognosis of advanced cancer patients. Italian Multicenter Study Group on Palliative Care. Cancer 2000;89:1145-1149.

21. Hui D, Kilgore K, Fellman B, et al.: Development and cross-validation of the in-hospital mortality prediction in advanced cancer patients (IMPACT) score: A preliminary study. J Palliat Med 2012;15:902-909.

22. Morita T, Tsunoda J, Inoue S, et al.: The palliative prognostic index: A scoring system for survival prediction of terminally ill cancer patients. Support Care Cancer 1999;7: 128-133.

23. Scarpi E, Maltoni M, Miceli R, et al.: Survival prediction for terminally ill cancer patients: Revision of the palliative prognostic score with incorporation of delirium. Oncologist 2011;16:1793-1799.

24. Knaus W, Draper E, Wagner D, et al.: APACHE II: A severity of disease classification system. Crit Care Med 1985;13:818-829.

25. Crawford GB, Agar MM, Quinn SJ, et al.: Pharmacovigilance in hospice/palliative care: Net effect of haloperidol for delirium. J Palliat Med 2013;16:1335-1341.

26. Currow DC, Rowett D, Doogue M, et al.: An international initiative to create a collaborative for pharmacovigilance in hospice and palliative care clinical practice. J Palliat Med 2012;15:282-286.

27. National Cancer Institute: Common terminology criteria for adverse events (CTCAE) version 4. Secondary common terminology criteria for adverse events (CTCAE) version 4 2009. http://ctep.cancer.gov/protocolDevelopment/electronic applications/ctc.htm- ctc_40. (Last accessed September 15, 2015).

28. Abernethy AP, Shelby-James T, Fazekas BS, et al.: The Australia-modified Karnofsky Performance Status (AKPS) scale: A revised scale for contemporary palliative care clinical practice [ISRCTN81117481]. BMC Palliat Care 2005;4:7.

29. Charlson ME, Pompei P, Ales K, et al.: A new method of classifying prognostic comorbidity in longitudinal studies: Development and validation. J Chronic Dis 1987;40:373-383.

30. de Groot V, Beckerman H, Lankhorst GJ, et al.: How to measure comorbidity. A critical review of available methods. J Clin Epidemiol 2003;56:221-229.

31. Meagher DJ, Trzepacz PT: Motoric subtypes of delirium. Semin Clin Neuropsychiatry 2000;5:75-85. 
32. O'Keeffe ST, Lavan JN: Clinical significance of delirium subtypes in older people. Age Ageing 1999;28:115-119.

33. Spiller JA, Keen JC: Hypoactive delirium: Assessing the extent of the problem for inpatient specialist palliative care. Palliat Med 2006;20:17-23.

34. Maust DT, Kim HM, Seyfried LS, et al.: Antipsychotics, other psychotropics, and the risk of death in patients with dementia: Number needed to harm. JAMA Psychiatry 2015;72:438-445.

35. Leonard MM, Agar M, Spiller JA, et al.: Delirium diagnostic and classification challenges in palliative care: Subsyndromal delirium, comorbid delirium-dementia, and psychomotor subtypes. J Pain Symptom Manage 2014;48:199-214.

(c) $(9 \ominus$ This work is licensed under a Creative Commons Attribution-NonCommercialBY No ND NoDerivatives 4.0 International License 